\title{
Comunicación
}

\section{Ectoparásitos en aves del género Agapornis en cautiverio en La Habana, Cuba}

\author{
Ectoparasites in birds of the genus A gapornis in CAPTIVIty in Havana, Cuba \\ Yenisey García Ferrer ${ }^{1,3}$, Daisy Rodríguez García ${ }^{1}$, Yanaisy Pino Quintana ${ }^{2}$
}

\section{Resumen}

El presente estudio tuvo como objetivo determinar la extensidad e intensidad de invasión de ectoparásitos que afectan a aves del género Agapornis criadas en cautiverio. Se trabajó con 50 aves de 8-9 meses, procedentes de tres criaderos de La Habana, Cuba. Se colectaron las plumas remeras de ambas alas. Se encontraron los ácaros plumícolas Pterophagus spp, Dubininia melopsittaci, ácaros de la superfamilia Pterolichoidea y el piojo malófago de la familia Philopteridae. La asociación de Pterophagus spp y el ácaro de la superfamilia Pterolichoidea presentó la mayor frecuencia. Los parásitos de mayor extensidad de invasión fueron Pterophagus spp y ácaros de la superfamilia Pterolichoidea. La intensidad de invasión fue muy leve para el piojo de la familia Philopteridae, con respecto a los ácaros, que se encontraron con niveles desde muy leve hasta muy grave.

Palabras clave: $\quad$ Agapornis; Pterophagus; Dubininia melopsittaci; Pterolichoidea; Philopteridae

\section{Abstract}

The objective of this study was to determine the extent and intensity of ectoparasite load affecting birds bred in captivity of the genus Agapornis. Fifty birds aged 8-9 months, from three hatcheries in Havana, Cuba were sampled. Feathers of both wings were

${ }^{1}$ Laboratorio de Investigaciones y Diagnóstico Aviar (LIDA) «Jesús Menéndez», Santiago de las Vegas, La Habana, Cuba

${ }^{2}$ Clínica «Tocororo» de la Filial La Habana de la Asociación Nacional Ornitológica de Cuba (ANOC), entre San Rafael e Infanta, Centro Habana, La Habana, Cuba

${ }^{3}$ E-mail: raul.montesino@informed.sld.cu 
collected. The mites Pterophagus spp, Dubininia melopsittaci, mites of the Pterolichoidea superfamily and the louse of the Philopteridae family were found. The association of Pterophagus spp and the mite of the superfamily Pterolichoidea presented the highest frequency. The most extensively invasive parasites were Pterophagus spp and mites of the Pterolichoidea superfamily. The intensity of invasion was slight for the louse of the family Philopteridae, with respect to mites, which were found with levels from very mild to very severe.

Key words: Agapornis; Pterophagus; Dubininia melopsittaci; Pterolichoidea; Philopteridae

\section{INTRODUCCIÓN}

Las psitácidas son aves que se comercializan por todo el mundo debido a la belleza de sus plumas y su don de articular palabras; sin embargo, al encontrarse en cautividad, con pérdida de su hábitat natural, pueden presentar diversos tipos de enfermedades. En Cuba, en particular, son frecuentemente afectadas por ectoparásitos, sobre todo, si conviven varias especies en un espacio reducido (Soto, 2010). Los artrópodos que afectan a las aves comprenden una gran diversidad de especies, entre ellos insectos y ácaros parasitiformes. Algunos juegan un papel importante como causantes de enfermedades al hombre, animales domésticos y silvestres, actuando como vectores biológicos y mecánicos de agentes patógenos, además de los daños directos que ocasionan por sus hábitos alimenticios y de fijación en diferentes partes del cuerpo (Parra-Henao et al., 2011).

Las aves del género Agapornis criadas en cautiverio pueden verse aquejadas por un número considerable de ectoparásitos. Es así que todo aquel que disponga de varios ejemplares de estas aves debe reducir el riesgo de parasitosis que se cierne sobre ellos y tener plena conciencia de situaciones que faciliten su propagación (Laufer et al., 2015).

Aunque ciertos criterios de manejo pueden ser extrapolados de otras especies, generalmente las parasitosis específicas resultan poco conocidas y pueden provocar pro- blemas en los aviarios (Larramendy et al., 2014). Algunos parásitos externos como los artrópodos no necesariamente se asocian con mermas en su desarrollo ni con la transmisión de enfermedades, pero pueden causar pérdidas económicas por daños en las plumas, aspecto esencial en la comercialización de estas aves (Laufer et al., 2015). Debido a los daños que pueden causar estos artrópodos, el presente estudio tuvo como objetivo determinar la proporción de aves Agapornis criadas en aviarios que son afectadas por ectoparásitos, así como el grado e intensidad del ectoparasitismo.

\section{Materiales y Métodos}

El experimento se realizó entre octubre y diciembre de 2016, con 50 aves ornamentales del género Agapornis criadas en cautiverio. Las aves, de ambos sexos, fueron seleccionadas al azar y con edades entre 8 y 9 meses. De ellas, 20 aves pertenecían al Parque Ecológico Medioambiental «Quinta de los Molinos», Oficina del Historiador, en el municipio Plaza de la Revolución, 10 procedían de un aviario asociado a la Clínica «Tocororo» de la Filial La Habana de la Asociación Nacional Ornitológica de Cuba (ANOC), municipio Centro Habana (privado A) y las otras 20 aves eran parte de un aviario privado en el municipio Playa, La Habana, Cuba (privado B). En el aviario de la Quinta de los Molinos se contaba con un total de 60 Agapornis y una masa de 300 aves, en el del privado A 
Cuadro 1. Condiciones de tenencia, manejo y alimentación de las aves

\begin{tabular}{|c|c|c|c|}
\hline $\begin{array}{l}\text { Tenencia, manejo y } \\
\text { alimentación }\end{array}$ & Quinta de los Molinos & Privado A & Privado B \\
\hline $\begin{array}{l}\text { Ubicación del } \\
\text { criadero }\end{array}$ & Orientación al norte & $\begin{array}{l}\text { Orientación al } \\
\text { norte }\end{array}$ & Orientación al norte \\
\hline Área de cuarentena & No presenta & No presenta & No presenta \\
\hline $\begin{array}{l}\text { Higiene de las } \\
\text { jaulas }\end{array}$ & $\begin{array}{l}\text { Limpieza de los comederos, } \\
\text { bebederos, pisos y rejillas a } \\
\text { diario o en días alternos y una } \\
\text { limpieza mecánica y húmeda } \\
\text { más profunda una vez a la } \\
\text { semana. No recicla los nidos }\end{array}$ & \multicolumn{2}{|c|}{$\begin{array}{l}\text { Limpieza con agua una sola vez a la } \\
\text { semana o cada dos semanas. Cambia los } \\
\text { nidos }\end{array}$} \\
\hline Desinfecciones & $\begin{array}{l}\text { Desinfección con compuestos } \\
\text { solubles en agua una vez a la } \\
\text { semana y un tiempo de reposo } \\
\text { de una semana }\end{array}$ & \multicolumn{2}{|c|}{$\begin{array}{l}\text { Desinfección cada tres meses y con el } \\
\text { producto que dispongan. Periodo de } \\
\text { reposo de tres días }\end{array}$} \\
\hline $\begin{array}{l}\text { Presencia de malla } \\
\text { anti-pájaros }\end{array}$ & \multicolumn{3}{|c|}{ Malla milimétrica o malla más gruesa y metálica en buen estado } \\
\hline $\begin{array}{l}\text { Control del } \\
\text { personal ajeno }\end{array}$ & $\begin{array}{l}\text { Restringe el ingreso de personas } \\
\text { ajenas al criadero. No cumple } \\
\text { las medidas de seguridad }\end{array}$ & \multicolumn{2}{|c|}{$\begin{array}{l}\text { Libre tránsito del personal ajeno. No } \\
\text { cumple las medidas de seguridad }\end{array}$} \\
\hline $\begin{array}{l}\text { Presencia de aves } \\
\text { silvestres }\end{array}$ & \multicolumn{2}{|c|}{ No cría aves silvestres dentro del mismo aviario } & $\begin{array}{l}\text { Aves silvestres dentro } \\
\text { del mismo aviario, pero } \\
\text { separadas de los } \\
\text { Agapornis }\end{array}$ \\
\hline $\begin{array}{l}\text { Control de } \\
\text { ectoparásitos }\end{array}$ & \multicolumn{2}{|c|}{$\begin{array}{l}\text { Desparasitación 2-3 veces en el año. Última hace } \\
4 \text { meses }\end{array}$} & $\begin{array}{l}\text { Desparasita una vez al } \\
\text { año. Última hace } 6 \\
\text { meses }\end{array}$ \\
\hline $\begin{array}{l}\text { Examen externo de } \\
\text { las plumas }\end{array}$ & $\begin{array}{l}\text { Examina } 10-20 \text { aves cada mes } \\
\text { para detectar ácaros y piojos }\end{array}$ & \multicolumn{2}{|c|}{$\begin{array}{l}\text { Examina }<10 \text { aves a simple vista cada dos } \\
\text { meses para detectar ácaros y piojos }\end{array}$} \\
\hline Alimentación & \multicolumn{2}{|c|}{$\begin{array}{l}\text { Variedad de semillas, aporte de suplementos } \\
\text { proteicos (blando) y suministro variado de } \\
\text { vegetales semanal }\end{array}$} & $\begin{array}{l}\text { Empleo de } 2-3 \text { semillas, } \\
\text { blando y suministro de } \\
\text { pocos vegetales mensual }\end{array}$ \\
\hline
\end{tabular}

con 30 Agapornis y una masa de 100 aves y en el del privado B con 50 Agapornis y 250 aves en total. Las otras especies presentes correspondieron mayormente a canarios (Serinus canaria) y pericos australianos (Melopsittacus undulatus).

Se analizaron las condiciones higiénicosanitarias de los aviarios, tomando en consideración los aspectos indicados en el Cuadro
1. Para el muestreo, las aves fueron sujetadas, extendiendo sus alas con cautela y cortando con tijeras estériles de tres a cinco plumas remeras de las alas (derecha e izquierda) a un centímetro por encima de la base de la pluma. Las muestras se colectaron en frascos estériles y se remitieron al Laboratorio de Investigaciones y Diagnóstico Aviar (LIDA) «Jesús Menéndez» del Instituto de Investigaciones Avícolas (IIA), en el munici- 
Cuadro 2. Clasificación de la intensidad de invasión según el número de piojos y ácaros de aves en plumas

\begin{tabular}{lcc}
\hline $\begin{array}{l}\text { Intensidad de } \\
\text { invasión }\end{array}$ & $\begin{array}{c}\text { Número de } \\
\text { piojos por } \\
\text { pluma }\end{array}$ & $\begin{array}{c}\text { Número de } \\
\text { ácaros por } \\
\text { pluma }\end{array}$ \\
\hline Negativo & 0 & 0 \\
Muy leve & $1-3$ & $1-5$ \\
Leve & $4-10$ & $6-25$ \\
Media & $11-25$ & $26-50$ \\
Grave & $26-50$ & $51-100$ \\
Muy grave & +50 & +100 \\
\hline
\end{tabular}

pio Boyeros, provincia La Habana. Las muestras fueron conservadas a $8{ }^{\circ} \mathrm{C}$ para evitar la fuga de los artrópodos y obtener falsos diagnósticos.

Los parásitos obtenidos fueron inmersos en solución de Hoyer y se montaron en láminas portaobjetos y se cubrieron con láminas cubreobjetos. Las muestras fueron incubadas a $28^{\circ} \mathrm{C}$ durante siete días para la clarificación. Posteriormente, las muestras fueron observadas en el estereoscopio y microscopio óptico para su identificación, teniendo en cuenta las estructuras básicas de los parásitos, según claves descritas por Gaud y Atyeo (1996) y Kranntz y Walter (2009).

Se determinó la extensidad de invasión (E.I.) de los ectoparásitos, mediante la fórmula propuesta por Roque (2015): E.I. $=($ Cantidad de animales positivos) / (total de animales) x 100. Asimismo, la intensidad de invasión (I.I.) o nivel de infestación se determinó contando la cantidad de ectoparásitos encontrados en cada pluma. Los criterios de valoración de la I.I. se efectuaron según la metodología señalada por Rodríguez et al. (2015) (Cuadro 2).

Se hizo un análisis de comparación de proporciones a los valores de la extensidad- de invasión, intensidad de invasión y a las asociaciones de ectoparásitos, así como la frecuencia de aves positivas a ectoparásitos.

\section{Resultados y Discusión}

Se diagnosticaron cuatro tipos de ectoparásitos en aves del género Agapornis en cautiverio, siendo los ácaros plumícolas Pterophagus spp, Dubininia melopsittaci, un ácaro de la superfamilia Pterolichoidea y un piojo malófago de la familia Philopteridae (Figura 1).

Los ácaros plumícolas hallados coinciden con los reportes de San Martín et al. (2005) y Ortiz et al. (2014), quienes los consideran de pequeño tamaño y se localizan en la superficie de las plumas, alimentándose de fragmentos de las plumas, grasas, detritus epiteliales y esporas de hongos.

El ácaro Dubininia melopsittaci, pese a que fue notificado por primera vez en Cuba en 2006 (Larramendy et al., 2007), es poco conocido por los parasitólogos. Este ectoparásito es específico de Melopsittacus undulatus, pero se le ha encontrado afectando a otras psitácidas en cautiverio como las aves del género Agapornis, cuando cohabitan juntos en un espacio reducido (Larramendy et al., 2014; Ortiz et al., 2014).

Las características morfológicas del piojo malófago de la familia Philopteridae concuerdan con las señaladas por González (2011). Es un piojo de gran tamaño, visible, tiene la cabeza más ancha que el tórax y posee un potente aparato masticador para la trituración de plumas y detritus celulares de la piel.

En el Cuadro 3 se aprecia que Pterophagus spp y los ácaros de la superfa-milia Pterolichoidea se diagnosticaron en una elevada proporción respecto a Dubininia melopsittaci y al piojo de la familia Philopteridae $(p<0.001)$. Este comportamiento 

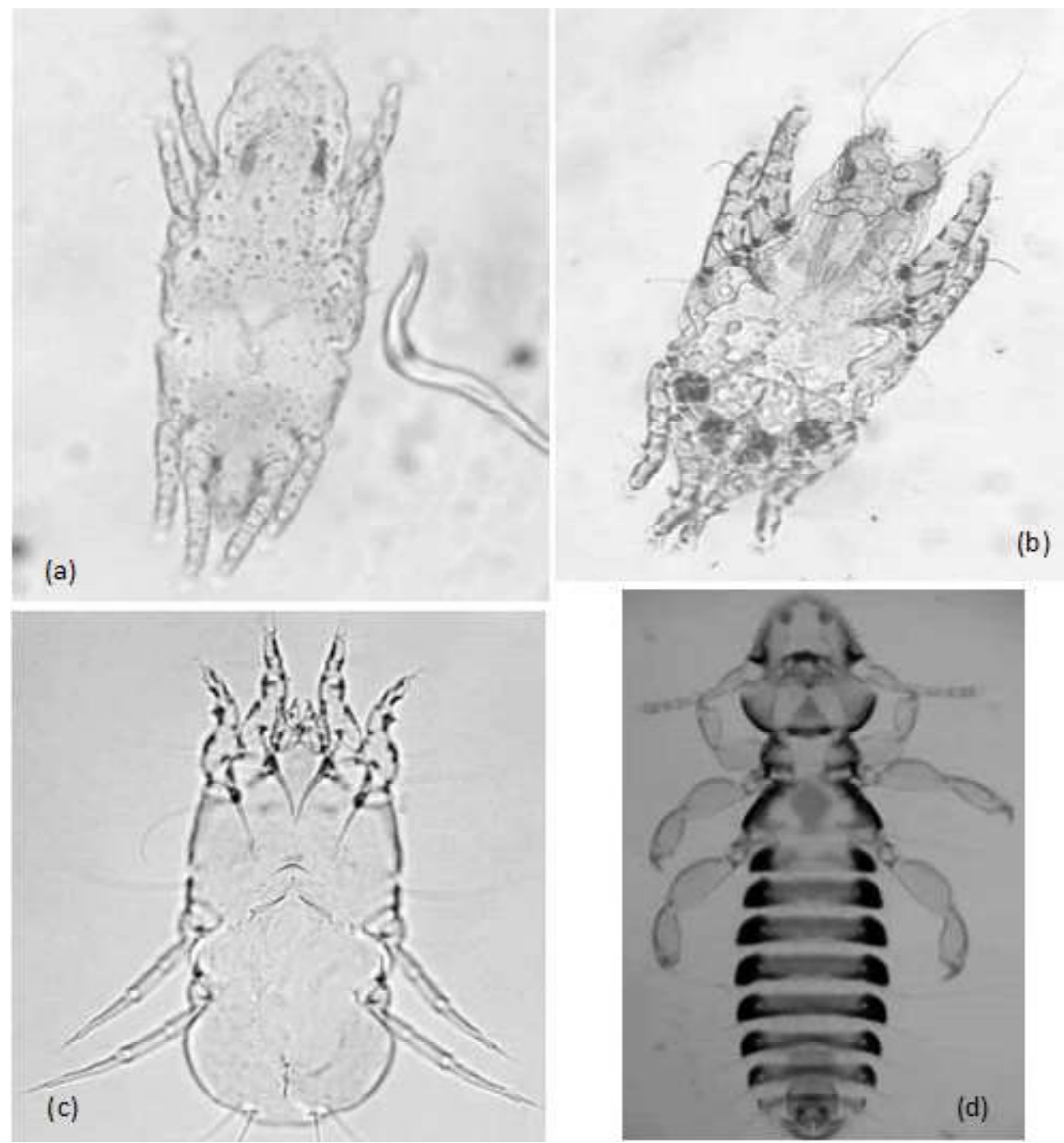

Figura 1. Ectoparásitos hallados en aves del género Agapornis criadas en cautiverio en La Habana, Cuba. a) Pterophagus spp; b) Superfamilia Pterolichoidea; c) Dubininia melopsittaci; d) Familia Philopteridae

coincide con los hallazgos de Larramendy et al. (2014).

La alta extensidad de los ectoparásitos constituye una situación alarmante, debido al estrés que causan en las aves (Sánchez, 2004; Hernández et al., 2007), ocasionando intranquilidad, reducción del consumo de alimentos y de hasta $15 \%$ de la producción de huevos; además se presenta un aumento en la producción de corticoesteroides y mayor susceptibilidad a las infecciones (Sánchez, 2004).
La baja E.I. del ácaro Dubininia melopsittaci pudo estar dada por las condiciones adversas en los aviarios que afectaron su supervivencia, tales como la escasa humedad, limpieza y desinfección periódica, así como la falta de adaptación a este hospedero. San Martín et al. (2005) y Valdebenito et al. (2015) consideran que los ácaros presentan una mayor prevalencia en otoño e invierno, debido a factores predisponentes, como la muda de las plumas, mayores temperaturas ambientales y humedad. Por otro 
Cuadro 3. Comparación de proporciones de la extensidad (E.I.) de invasión de ectoparásitos en 50 aves del género Agapornis criadas en cautiverio en La Habana, Cuba

\begin{tabular}{llccc}
\hline \multirow{2}{*}{ Ectoparásitos } & $\begin{array}{c}\text { Aves afectadas } \\
(\mathrm{n})\end{array}$ & Proporción & $\begin{array}{c}\text { E.I. } \\
(\%)\end{array}$ \\
\hline \multirow{2}{*}{ Ácaros } & Pterophagus spp & 36 & 0.72 & $72^{\mathrm{a}}$ \\
& Superfamilia Pterolichoidea & 27 & 0.54 & $54^{\mathrm{a}}$ \\
& Dubininia melopsittaci & 5 & 0.10 & $10^{\mathrm{b}}$ \\
Piojo & Familia Philopteridae & 1 & 0.02 & $2^{\mathrm{b}}$ \\
\hline
\end{tabular}

Comparación de proporciones: Prueba $\mathrm{F}=25.39 * * *(E S=0.07)$

a,b Valores con letras diferentes dentro de columnas son significativamente diferentes $(p<0.001)$

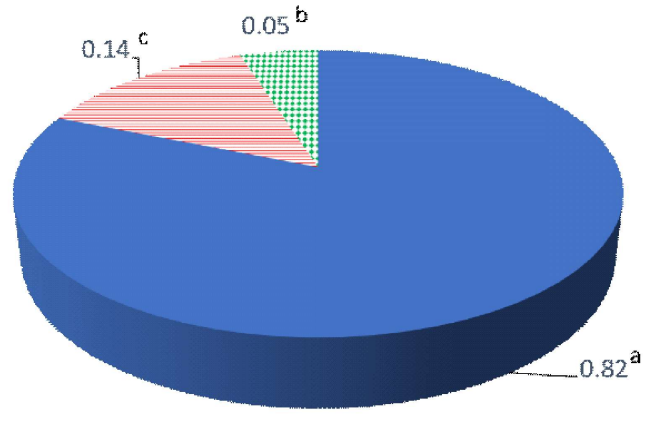

Pterophagus spp + Superfamilia Pterolichoidea

$\equiv$ Pterophagus spp + Dubininia melopsittaci

:Pterophagus spp + Familia Philopteridae

Figura 2. Proporciones de las asociaciones de ectoparásitos en aves del género Agapornis criadas en cautiverio en La Habana, Cuba. Comparación de proporciones: Prueba $\mathrm{F}=17.66 * * *(\mathrm{ES}=0.10)$. Valores con letras diferentes son significativamente diferentes $(\mathrm{p}<0.001)$

lado, la presencia del piojo de la familia Philopteridae en una sola ave no coincide con los hallazgos de Saavedra et al. (2014), quienes plantean que estos parásitos tienen un mayor desarrollo durante los meses más fríos.

Es importante destacar la zona de toma de las muestras de plumas. Wall y Shearer (2001) señalan que la mayoría de las especies de piojos tienen una alta especificidad de hospederos y muchos se especializan en parasitar solo una parte del cuerpo del ave. Así mismo, Saavedra et al. (2014) plantean que las variaciones de microclima entre las plumas y de temperatura en distintas partes del cuerpo podrían reducir la sobrevivencia de los piojos, delimitando los mismos a determinadas zonas de su hospedero. Lo anterior ratifica la necesidad de tomar plumas de diferentes porciones del cuerpo para lograr llegar a un diagnóstico certero.

En el resultado de las asociaciones de ectoparásitos en las aves, se observa que Pterophagus spp y el ácaro de la superfamilia Pterolichoidea se encontraron en mayor proporción, con diferencias altamente significativas, respecto al resto de las combinaciones parasitarias que mostraron menores proporciones (Figura 2). Las asociaciones de ectoparásitos con presencia de Pterophagus spp se observó en 22 aves, indicando su gran adaptación a la convivencia con otras especies.

No se pudo llegar a diagnosticar la especie del ácaro Pterophagus sp, superfamilia Pterolichoidea y piojo de la familia Philopteridae, dado que no es alguna de las descritas en el país y todavía no se dispone de las claves taxonómicas para su identificación; sin embargo, Galloway et al. (2014) explican que la superfamilia Pterolichoidea comprende nueve familias de ácaros, siendo 


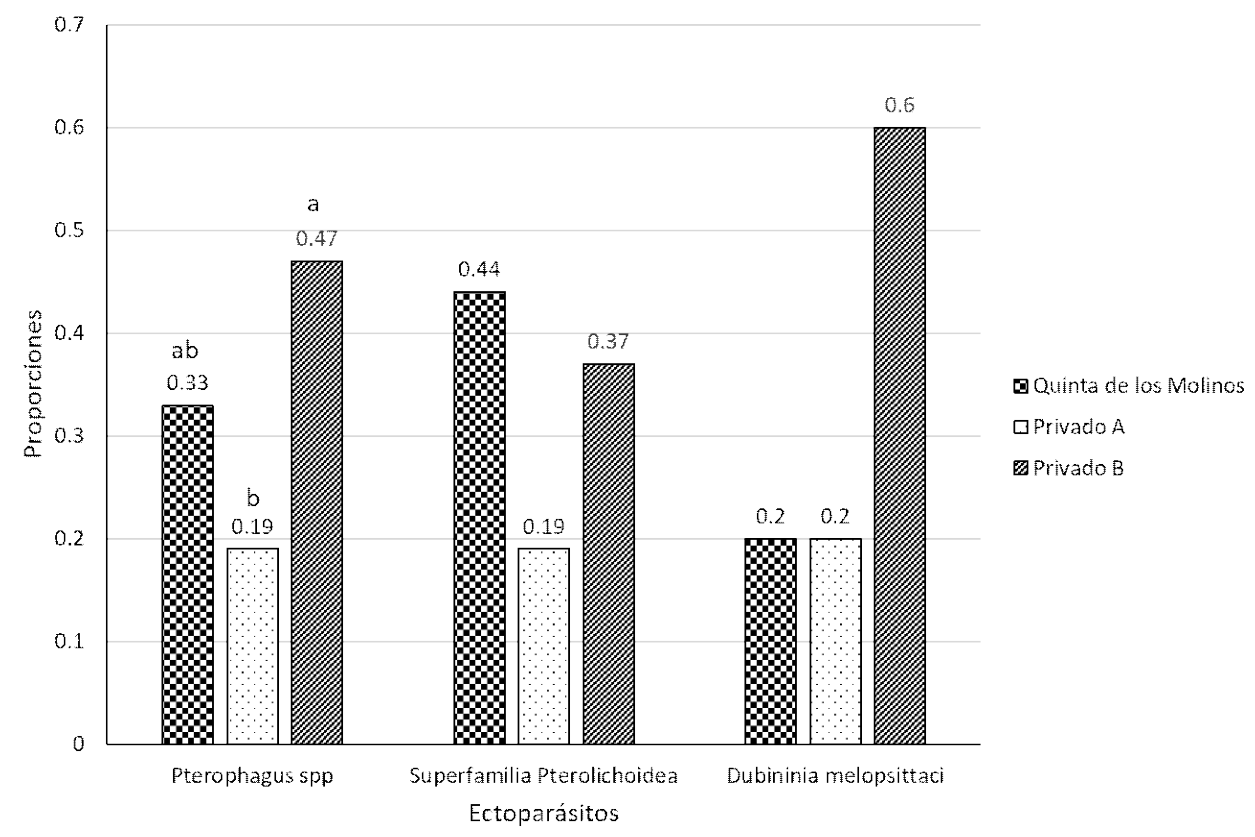

Figura 3. Comparación de proporciones de las aves positivas a ectoparásitos del género Agapornis criadas en cautiverio en tres aviarios de La Habana, Cuba. Valores con letras diferentes dentro de grupos de parásitos son estadísticamente diferentes $(\mathrm{p}<0.05)$

Cuadro 4. Comparación de proporciones (prop.) de la intensidad de invasión (I.I.) de ectoparásitos en 50 aves del género Agapornis criadas en cautiverio en La Habana, Cuba

\begin{tabular}{|c|c|c|c|c|c|c|c|c|}
\hline \multirow{3}{*}{$\begin{array}{l}\text { Intensidad de } \\
\text { invasión }\end{array}$} & \multicolumn{6}{|c|}{ Ácaros } & \multirow{2}{*}{\multicolumn{2}{|c|}{$\begin{array}{c}\text { Piojo } \\
\text { Familia } \\
\text { Philopteridae }\end{array}$}} \\
\hline & \multicolumn{2}{|c|}{ Pterophagus spp } & \multicolumn{2}{|c|}{$\begin{array}{c}\text { Superfamilia } \\
\text { Pterolichoidea }\end{array}$} & \multicolumn{2}{|c|}{$\begin{array}{c}\text { Dubininia } \\
\text { melopsittaci }\end{array}$} & & \\
\hline & $\begin{array}{l}\text { Aves } \\
\text { (n) }\end{array}$ & Prop. & $\begin{array}{l}\text { Aves } \\
\text { (n) }\end{array}$ & Prop. & $\begin{array}{l}\text { Aves } \\
\text { (n) }\end{array}$ & Prop. & $\begin{array}{c}\text { Aves } \\
\text { (n) }\end{array}$ & Prop. \\
\hline Muy leve & 6 & 0.17 & 5 & 0.19 & 1 & 0.20 & 1 & 1.00 \\
\hline Leve & 9 & 0.25 & 7 & 0.26 & 3 & 0.60 & - & - \\
\hline Media & 12 & 0.33 & 4 & 0.15 & 1 & 0.20 & - & - \\
\hline Grave & 9 & 0.25 & 8 & 0.30 & - & - & - & - \\
\hline Muy grave & - & - & 3 & 0.11 & - & - & - & - \\
\hline Total (n) & \multicolumn{2}{|c|}{36} & \multicolumn{2}{|c|}{27} & \multicolumn{2}{|c|}{5} & \multicolumn{2}{|c|}{1} \\
\hline
\end{tabular}

No significativo $(p>0.05)$ 
la familia Pterolichidae la más diversa con cerca de 100 géneros, donde más de 20 géneros afectan a Psittaciformes.

La intensidad de invasión de los ectoparásitos no fue estadísticamente diferente entre ellos (Cuadro 4), lo que indica que las invasiones se encontraron a cualquier nivel, aunque solo se interpretan como problema cuando la intensidad es de media a muy grave, donde los daños en las plumas suelen ser muy marcados.

Los resultados coinciden con lo planteado por Romero (2001) y San Martín et al. (2005), quienes consideran que algunos ácaros no causan efectos evidentes a menos que su población sea grande, lo cual podría ser detectado al observar el plumaje sucio, quebrado y áspero. Las invasiones clínicas de ectoparásitos llevan a la caída de las plumas, además de producir intranquilidad, arreglo excesivo y frenético (Sánchez, 2004; Hernández et al., 2007). Sin embargo, a pesar de que no se apreciaron diferencias significativas, es necesario tener un control de las poblaciones parasitarias para evitar una alta intensidad de invasión. Soto y Bert (2012) refieren que la posibilidad de que un ave ornamental pueda tener ectoparásitos siempre puede estar latente. Por otro lado, Saavedra et al. (2014) resaltan la importancia de tener un registro del tipo de ectoparásito involucrado en caso de haber infestaciones severas.

En la Figura 3 se aprecian que el ácaro Pterophagus spp se encontró en una mayor y significativa proporción en el aviario del privado B respecto al aviario de Quinta de los Molinos $(\mathrm{p}<0.05)$, no existiendo diferencias significativas para los demás parásitos entre los tres aviarios en estudio.

\section{Conclusiones}

- Los ectoparásitos de mayor extensidad de invasión fueron Pterophagus spp y ácaros de la superfamilia Pterolichoidea.
- La intensidad de invasión fue muy leve para el piojo de la familia Philopteridae, con respecto a Pterophagus spp y ácaros de la superfamilia Pterolichoidea y Dubininia melopsittaci que se encontraron con niveles desde muy leve hasta muy grave.

\section{Literatura Citada}

1. Galloway T, Proctor H, Mironov S. 2014. Chewing lice (Insecta: Phthiraptera: Amblycera, Ischnocera) and feather mites (Acari: Astigmatina: Analgoidea, Pterolichoidea): Ectosymbionts of grassland birds in Canada. In: Arthropods of Canadian grasslands: biodiversity and systematics. Manitoba, Canada: Biological Survey of Canada. p 139-188.

2. Gaud J, Atyeo W. 1996. Feather mites of the world (Acarina: Astigmata): the supraspecific taxa. Belgium: Koninklijk Museum voor Midden-Afrika. $436 \mathrm{p}$.

3. González S. 2011. Parasitología na medicina veterinária. Brasil: Roca. $370 \mathrm{p}$.

4. Hernández $M$, Szczypel $B$, Larramendy R, Temprana M, Ramos M, Miranda I. 2007. Dinámica de la población parasitaria: Megninia ginglymura (Acari: Analgidae): criterios de modelación. Rev Cubana Cienc Avíc 31(2): 127-134.

5. Kranntz G, Walter D. 2009. A manual of acarology. $3^{\text {rd }}$ ed. Texas. $598 \mathrm{p}$.

6. Larramendy $R$, Hernández $B$, Szczypel M, Temprana I, Morales Y, Ramos M, Cuervo N. 2007. Parásitos internos y externos diagnosticados en pequeñas psitácidas de compañía. Informe de Dubininia melopsittaci (Acari: Xolalgidae) en Cuba. Rev Cubana Cienc Avíc 34: 89-96.

7. Larramendy R, Rodríguez D, García, M, Cuervo N, López J, Reinaldo O, Xuárez M, Morales Y, Rebollar R. 2014. Ácaros plumícolas en aves ornamentales: primer informe de Nymphi-cilichus perezae (Acari: Pterolichidae) para Cuba. Rev Cubana Cienc Avíc 38: 65-67. 
8. Laufer G, Grosso E, Smith S. 2015. Piojos masticadores de plumas, ectopará-sitos del ñandú (Rhea americana). VI Congreso Internacional sobre Manejo de Fauna Silvestre en Amazonía y Latinoamérica. Iquitos, Perú.

9. Ortiz R, Muñoz C, Rendón E, Acosta R, Montiel G. 2014. Artrópodos asociados a las aves de la familia Anatidae del estado de Tlaxcala, México. Entomol Mexicana 1: 458-463.

10. Parra-Henao G, Alarcón E, López G, Ramírez D, Jaramillo G. 2011. Detección de ectoparásitos en aves silvestres evaluadas en Medellín (Colombia). Rev Colomb Cienc Pecu 24(1): 29-37.

11. Rodríguez D, Larramendy R, Varona E, Colas M, Reinaldo O, Villa J, Morales Y, Cobas I, Rebollar R. 2015. Comportamiento de los parásitos externos en aves (Gallus gallus) de diferentes líneas puras. Rev Cubana Cienc Avíc 39(2): 5-12.

12. Romero E. 2001. Enfermedades más comunes de las codornices. México. [Internet]. Disponible en: http:// www.agrobit.com.ar/Microemprendimientos/cria_animales/avicultura/ MI000003av.htm

13. Roque E. 2015. Parasitología y enfermedades parasitarias en los animales domésticos. La Habana: Félix Varela. 322 p.

14. Saavedra A, Arévalo S, Soler D. 2014. Ectoparásitos del orden Phthiraptera en aves silvestres. Mem Conf Interna Aprovech Fauna Silv Exot Conv 10(2): 5-24.
15. San Martín J, Brevis C, Rubilar L, Schmäschke R, Daugschies A, González-acuña D. 2005. Ectoparasitismo en tiuque común Milvago chimango chimango (Vieillot, 1816) (Aves, Falconidae) en la zona de Ñuble, Chile. Lundiana 6(1):49-55

16. Sánchez A. 2004. Principales enfermedades que afectan a las aves. En: Sánchez A, López A, Sardá R, Pérez M, et al. (eds). Salud y producción de las aves. La Habana: UNAH. p 185-465.

17. Soto C, Bert E. 2012. Valoración sanitaria de los criaderos de aves ornamentales. REDVET 13(7). [Internet]. Disponible en: http://www.veterinaria.org/ revistas/redvet/n070712/071221.pdf

18. Soto C. 2010. Las psitácidas cubanas y su preservación. REDVET 11(11B). [Internet]. Disponible en: http:// www.veterinaria.org/revistas/redvet/ n111110B/111006B.pdf

19. Valdebenito J, Moreno L, LandaetaAqueveque C, Mike J, Mironov S, Cicchino A, et al. 2015. Gastrointestinal and external parasites of Enicognathus ferrugineus and Enicognathus leptorhynchus (aves, Psittacidae) in Chile. Rev Bras Parasitol Vet 24: 422-31. doi: 10.1590/S1984-29612015074

20. Wall R, Shearer D. 2001. Veterinary ectoparasites: biology, pathology and control. Canada: Blackwell Science. 420 p. 